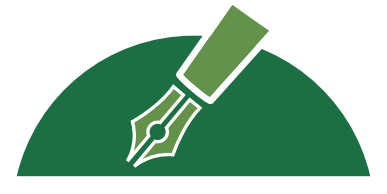

Lecturas críticas

Apropiación social del conocimiento

Generación de contenidos impresos

https://repository.ucc.edu.co/handle/20.500.12494/7375

N. ${ }^{\circ} 14$, diciembre de 2021

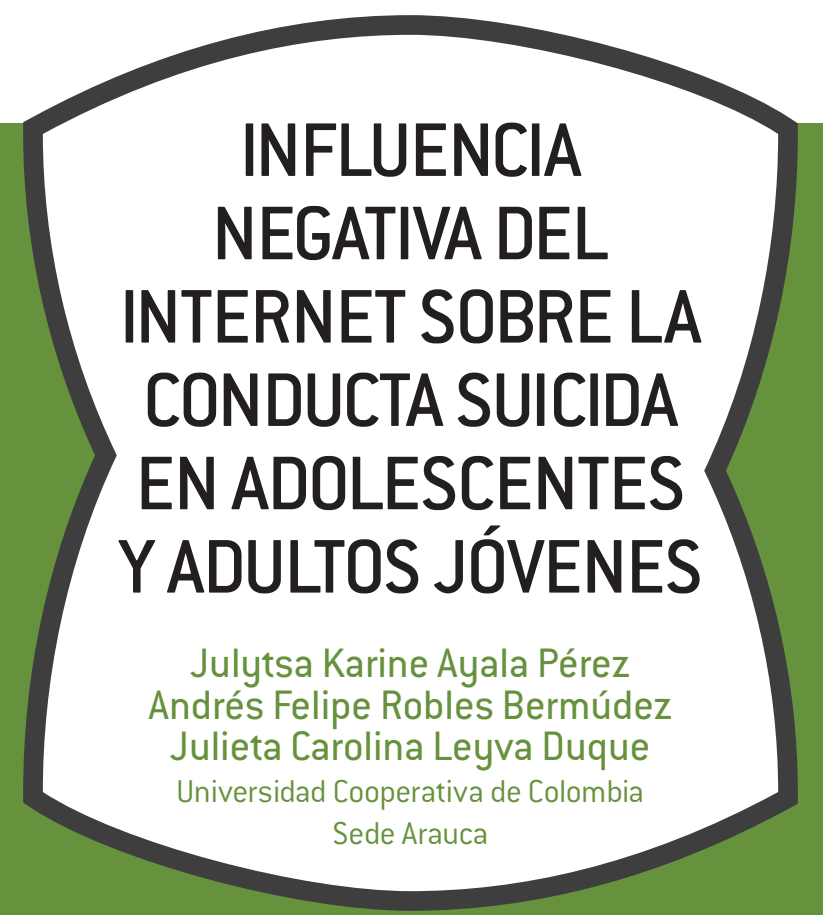

doi: https://doi.org/10.16925/gclc.20

\title{
NOTA LEGAL
}

El presente documento de trabajo ha sido incluido dentro de nuestro repositorio institucional como Apropiación social de conocimiento por solicitud del autor, con fines informativos, educativos o académicos. Asimismo, los argumentos, datos y análisis incluidos en el texto son responsabilidad absoluta del autor y no representan la opinión del Fondo Editorial o de la Universidad.

\section{DISCLAIMER}

This coursework paper has been uploaded to our institutional repository as Social Appropriation of Knowledge due to the request of the author. This document should be used for informational, educational or academic purposes only. Arguments, data and analysis included in this document represent authors' opinion not the Press or the University. 


\section{ACERCA DE LOS AUTORES}

Julytsa Karine Ayala Pérez, psicóloga, especialista en Docencia y Pedagogía, maestrante en Educación, docente de la Facultad de Ciencias Sociales, Programa de Psicología, Universidad Cooperativa de Colombia, sede Arauca.

Correo electrónico:

julytsa.ayalap@campusucc.edu.co

Andrés Felipe Robles Bermúdez, estudiante del programa de Psicología, Universidad Cooperativa de Colombia, Centro de Extensión de Arauca. Correo electrónico:

andres.roblesb@campusucc.edu.co

Julieta Carolina Leyva Duque, estudiante del programa de Psicología, Universidad Cooperativa de Colombia, Centro de Extensión de Arauca. Correo electrónico:

julieta.leyvad@campusucc.edu.co

\section{Cómo CITAR ESTE DOCUMENTO}

Ayala Pérez, J. K., Robles Bermúdez, A. F. y Leyva Duque, J. C. (2021). Influencia negativa del internet sobre la conducta suicida en adolescentes y adultos jóvenes (Generación de contenidos impresos N. 14). Ediciones Universidad Cooperativa de Colombia. https://doi.org/10.16925/gclc.20

Este documento puede ser consultado, descargado o reproducido desde nuestro repositorio institucional (http://repository. ucc.edu.co/handle/20.500.12494/7369) para uso de sus contenidos, bajo la licencia de Creative Commons Reconocimiento-NoComercial-SinObraDerivada 4.0 Internacional. http:// creativecommons.org/licenses/by-nc-nd/4.0/ 


\title{
14 INFLUENCIA NEGATIVA DEL 14 INTERNET SOBRE LA CONDUCTA SUICIDA EN ADOLESCENTES Y ADULTOS JÓVENES
}

\author{
Julytsa Karine Ayala Pérez \\ Andrés Felipe Robles Bermúdez \\ Julieta Carolina Leyva Duque
}

\section{Resumen}

En la actualidad, es fundamental prestar mayor atención al uso de la tecnología, ya que existe la preocupación por la influencia negativa que trae consigo el internet en relación con la conducta suicida en los adolescentes, ya que estos pasan gran parte de su tiempo en un dispositivo electrónico, algunos incluso sin ningún tipo de restricción. Para este trabajo, se realizó una búsqueda sistemática con énfasis en la revisión y el análisis de aquellos factores negativos del internet y la conducta suicida, utilizando fuentes de diferentes bases de datos que ayudaron a determinar teóricamente si el internet influye de manera negativa en la conducta suicida. De la revisión se pudo concluir que la mayoría de los jóvenes con comportamientos suicidas son vulnerables a la información que hay en internet, a tal punto que puede presentarse el riesgo de ejecución de acto suicida.

Palabras clave: adolescentes, cibersuicidio, factores de riesgo, internet, redes sociales. 


\section{TABLA DE CONTENIDO}

INTRODUCCIÓN

Planteamiento del Problema

$\begin{array}{ll}\text { REVISIÓN ACTUAL DE LA PROBLEMÁTICA } & 7\end{array}$

$\begin{array}{ll}\text { CONCLUSIÓN } & 7\end{array}$

REFERENCIAS $\quad 8$ 


\section{INTRODUCCIÓN}

La presente lectura crítica tiene como objetivo realizar una revisión sistemática acerca de la influencia negativa del internet sobre la conducta suicida. Para hacer posible en el lector una buena compresión de lo establecido en este documento, es importante identificar conceptos básicos y relaciones directas e indirectas del internet sobre el suicidio; para ello, se revisarán diferentes fuentes bibliográficas, bases de datos y estudios desarrollados con relación al tema principal.

Cerca de 26300000 resultados se encuentran con solo poner la palabra suicidio en un motor de buscada como Google. Toda esta información se divide en noticias, definiciones, cifras, conceptos teóricos, métodos, foros, etc.; los datos se alojan en páginas web que en su mayoría no tienen un control sobre quienes acceden, manipulan o distorsionan la información.

Con el propósito de establecer un punto de partida, cabe señalar que la conducta suicida es generada por un conjunto de situaciones por las que pasa un individuo y que muchas veces lo llevan a consumar el acto, atravesando por diversas fases: inicia con los pensamientos que conducen a la ideación (planificación) y culmina con el intento y realización con objetivo cumplido o no. Esto no quiere decir que los individuos con conducta suicida estén decididos a ejecutar el acto.

Ahora bien, dicha conducta se ha visto influenciada de manera exponencial por el uso desmedido del internet, lo que trae consigo nuevos términos y nuevos riesgos, como, por ejemplo, el cibersuicidio, que según Pérez (2019) "se refiere a la influencia de la información que circula en internet sobre el suicidio, así como [...] la incitación que hay en dichos medios para ejercerlo" (p. 4). De este modo, la población con mayor accesibilidad e interacción en internet son adolescentes y adultos jóvenes, que, en cifras mundiales, constituyen el conjunto más afectado.

Según la Organización Mundial de la Salud (OMs, 2019), "en promedio 800000 personas se quitan la vida cada año [...], cada suicidio es una tragedia que afecta a familias, comunidades y países [...], el suicidio se puede producir a cualquier edad, y en el 2016 fue la segunda causa principal de defunción en el grupo etario de 15 a 29 años en todo el mundo". Este fenómeno no ha dejado de preocupar a las entidades de salud pública o a la misma población, no solo por ser una problemática social vigente, sino también porque la conducta suicida es silenciosa y los mitos urbanos le restan importancia. Señala Mental Health Europe (2020) en este sentido:

Las creencias erróneas y el estigma asociados con el suicidio lo convierten todavía en un tema "tabú" para muchos. Por ello, la información y formación sobre este tema es fundamental. [...] Desmentir los mitos sobre el suicidio no solo reduce el estigma: ayuda a la sociedad a comprender que alguien está en riesgo y a buscar ayuda. (citado en Infocop, 2020)

A modo de conclusión, el internet es una herramienta ambivalente: así como sirve para obtener información clara y objetiva, también lo es para desinformar, con lo cual genera un factor de riesgo y un medio facilitador de la conducta suicida. La consecuencia es que el individuo vulnerable llegue más rápido y eficazmente a cometer el acto de quitarse la vida: se expone ante un caso o modelo a seguir, y con ello tiene lugar un contagio suicida.

\section{Planteamiento Del PROBLEMA}

El internet podría definirse como una red global que se interconecta de manera instantánea entre diferentes dispositivos, ya sean 
ordenadores, celulares, computadores, etc., y que facilita los procesos de información, comunicación e interacción. Los adolescentes y adultos jóvenes son quienes en su mayoría hacen uso de estas herramientas y páginas de interacción social, como lo son Google, Facebook, WhatsApp, etc. La adquisición de datos nunca había sido tan rápida y eficiente en toda la historia; no obstante, las horas en internet, la desinformación, las noticias falsas y las sugerencias de páginas web con información sensible acerca de la conducta suicida también se han convertido en factores de riesgo para la salud mental y física.

Al mismo tiempo, al realizar la búsqueda de cibersuicidio, en diferentes páginas web existe un fácil acceso y sin ningún tipo de restricción a información relacionada. Temas como net suicide, juegos pro suicidio, foros pro suicidio, ciberbullying, transmisiones de suicidio en vivo, métodos eficaces para consumar el acto y mucha más información latente está disponible para cualquier persona, sin importar edad o una adecuada salud mental.

Dentro del conjunto de conceptos intrínsecos del cibersuicidio, encontramos el net suicide, entendido por Molina y Restrepo (2018) como "espacios en línea que ofrecen oportunidades para compartir ideas y planes suicidas” (p. 36). Allí se socializan y estructuran ideas, planes y pactos suicidas entre dos o más individuos que acuerdan un lugar y un tiempo por medio de la internet. Además, están los juegos suicidas: por medio de retos progresivos y actos autolesivos, se conduce a los adolescentes a consumar el suicidio. Entre ejemplos de ellos, encontramos el reto de la ballena azul, momo, entre otros, algunos muy mencionados por los medios de comunicación; no obstante, existen muchos otros más que no son tan expuestos, pero que los niños y adolescentes pueden conocer con gran facilidad.

Adicionalmente, las redes sociales han abierto un mundo de riesgos latentes para las personas que navegan la red: se acepta una nueva normalidad sobre las interacciones con pares; ver cómo se ofenden, critican y atacan por ser diferentes a los demás es lo cotidiano entre adolescentes. Según Larrañaga et al. (2018), "la tendencia al uso de estos medios para emitir mensajes de texto, audios por el móvil y publicar fotografías y videos difundidos por las redes sociales, sin permiso de sus autores y con la intención de ridiculizar o con otros fines negativos, es conocido como ciberbullying" (p. 215).

Desde otro punto de vista, es importante mencionar que el avance tecnológico ha creado una brecha entre los programas de prevención y los factores de riesgo; por consiguiente, plataformas digitales como YouTube, Facebook Live, Instagram, entre otras, posibilitan el acceso a eventos en vivo, como los suicidios en línea. Se puede señalar el caso de Arslán Valéyev, un youtuber ruso que grabó en línea el método utilizado para quitarse la vida, dejando en evidencia sus últimos momentos. En todo caso, y con ayuda del presente ejercicio académico, no se ha encontrado evidencia científica que demuestre la existencia de programas preventivos con el fin de afrontar este comportamiento suicida en páginas pro suicidio. Estas últimas, según Martínez (2020), "son un intercambio de información de diferente contenido - pro suicidio o ayuda no profesional- que incita en la mayoría de casos a promover una atmósfera virtual de agresividad, insultos, la intimidación verbal o descalificación a la propia vida y circunstancias expuestas" (p. 32).

Por último, dentro del cibersuicidio se encuentran los métodos para ejecutar la conducta suicida; aunque no hay gravedad en el asunto de exponer la información relacionada con el suicidio con una base educativa, sí existe un riesgo, ya que no se revisa si la persona que está buscando dicha información presenta la ideación suicida con fines de planear cómo sería más fácil terminar con su propia vida. 


\section{REVISIÓN ACTUAL DE LA PROBLEMÁTICA}

En la actualidad, cuando pensamos o hacemos referencia a la comunicación con los pares y la búsqueda sistemática de información, nuestras acciones son direccionadas a hacer uso de un Smartphone, Tablet o MacBook para llevar a cabo este proceso, gracias a su accesibilidad. Hoy en día la interacción social de las personas se ve estrechamente relacionada con el ciberespacio, algo que "representa un cambio de vida en las personas, en especial para la población joven, ya que la mayor parte de ellos utiliza el internet y las redes sociales como medio de interacción social" (Domínguez et al., 2020, p. 5). Agregan Jung y Kim (2021) al respecto:

Más del 50\% del contenido con temas de suicidio en YouTube incluye ilustraciones gráficas, verbales o textuales de métodos de suicidio; sin embargo, la mayoría no proporcionó ninguna señal de advertencia o líneas directas de crisis en relación con la edad de la persona que accede a este tipo de información. [traducción propia]

De acuerdo con lo anterior, el contenido suicida que se encuentra en internet es accesible a todo tipo de población sin ningún grado de restricción. Indican Molina y Restrepo (2018):

El internet, un medio de comunicación ampliamente difundido y particularmente aceptado e incorporado por los adolescentes en su estilo de vida y de interacción consigo mismos y con otros, fue incorporado dentro de los factores de riesgo para el comportamiento suicida de jóvenes. (p. 37)

Al ser un medio de fácil accesibilidad y contenido no regulado, internet permite a los jóvenes proximidad a cualquier tipo de información e interacción social impersonal y deshumanizada acerca del cibersuicidio. Asimismo, en este contexto, se deben tener en cuenta otros factores como "las noticias o la cobertura televisiva de historias de suicidio, ya que estas pueden proporcionar modelos a seguir para las personas en riesgo, lo que está relacionado con una teoría del aprendizaje social de la conducta desviada” (Jung y Kim, 2021, p. 3). Las historias publicitadas por los medios de comunicación, transmitidas en vivo desde plataformas como Facebook o YouTube, pueden fomentar el suicidio en adolescentes vulnerables en el mundo real. Sostienen Picardo et al. (2020):

Existe una gran preocupación [de] que la cobertura extensa de suicidios reportados de manera sensacionalista o glamurosa [...] o dar detalles explícitos sobre métodos de autolesión, se asocie con un aumento de las conductas suicidas entre las personas vulnerables: un contagio o efecto "Werther". (p. 1)

En línea con lo anterior, Shanahan et al. (2019) concluyen que "académicos, médicos y otros han expresado su preocupación sobre si las redes sociales fomentan la autolesión, ya sea normalizando el comportamiento o conectando a los jóvenes con otras personas que participan en él y lo alientan" (p. 1).

\section{CONCLUSIÓN}

El internet y las redes sociales se han convertido en un medio rápido y eficaz de búsqueda de datos e interacción entre las personas, especialmente entre jóvenes. Sin embargo, dentro del contenido existente en la red, no se ha podido establecer si la información sirve para prevenir o promover problemáticas sociales como el suicidio. En este marco, la revisión sistemática de información permitió encontrar páginas antisuicidios que orientan a la prevención, pero a su vez se hallaron paginas pro suicidio que 
incentivan la conducta suicida. Aunque en esta ocasión, y desde la premisa de un trabajo académico, lo evidenciado sirve para contextualizar al lector, no hay que dejar a un lado la preocupante idea de personas con riesgo suicida utilizando estos sitios web para concretar la planeación de la conducta autolesiva.

De manera análoga, esto se vincula con la forma en que noticias, imágenes y textos acerca del acto suicida son presentadas al consumidor. Especialmente los adolescentes son susceptibles a ejecutar acciones autolesivas, como resultado del aprendizaje sobre el comportamiento suicida de otros; algo denominado también como contagio suicida, que "se propone como un efecto, a corto plazo, de la exposición a historias en la web de intentos de suicidio o suicidio consumado" (Molina y Restrepo, 2018, p. 34). Esto indudablemente llama la atención de aquellos que usan frecuentemente el internet como actividad de ocio.

En conclusión, los jóvenes pueden tener un riesgo latente ante el suicidio: se consideran una población vulnerable ante la información que hay en internet, aumentada por la falta de control en relación con el contenido plasmado en las páginas web; entonces, junto con el ejercicio académico, la respuesta al interrogante "¿el internet influye negativamente en la conducta suicida?" es ciertamente un rotundo sí.

\section{REFERENCIAS}

Domínguez Vargas, A. P., Rodríguez Rojas, D. P. y Torres Gutiérrez, A. G. (2020). Redes sociales en la interacción social (trabajo de grado en Psicología). Universidad Cooperativa de Colombia. https://bit.ly/3uya0o7

El Nacional. (2017, 27 de septiembre). Youtuber ruso se dejó morder por su serpiente en plena transmisión en vivo. https://bit.ly/2ZTB325

Galeano, S. (2021, 29 de enero). El número de usuarios de internet en el mundo crece un 7,3\% y alcanza los 4.660 millones. M4rketing Ecommerce. https://bit.ly/3D64kEK

Fernández, J. (2020). Digital 2020: el uso de las redes sociales abarca casi la mitad de la población mundial. We Are Social. https://bit.ly/3B1hbaP

Jung, E. J. y Kim, S. (2021). Suicide on YouTube: Factors engaging viewers to a selection of suicide-themed videos. PLoS One, 16(6), e0252796. https://doi.org/10.1371/journal.pone.0252796

Martínez, L. (2020). Suicidio, adolescencia, redes sociales e internet. https://bit.ly/3oq6POn

Infocop. (2020). En la prevención del suicidio es fundamental erradicar los mitos en torno al mismo, según Mental Health Europe. Infocop Online. https://bit.ly/3zVFp4U

Larrañaga, E., Navarro, R. y Yubero, S. (2018). Factores sociocognitivos y emocionales en la agresión del ciberacoso. Revista Científica de Comunicación y Educación, 26(56). https://doi.org/10.3916/C56-2018-02

López, E.J. M., Cuervo,Á.A.V., Murrieta, M. U.,Acuña, G. M.T.y Parra-Pérez, L. G. (2020). Factores asociados al ciberacoso en adolescentes. Perfiles Educativos, 42(167). https://doi.org/10.22201/iisue.24486167e.2019.167.59128

Lung, F., Shu, B., Chiang, T. y Lin, Sh. (2020). Relationships between internet use, deliberate self-harm, and happiness in adolescents: A Taiwan birth cohort pilot study. PLoS One 15(7), e0235834. https://doi.org/10.1371/ journal.pone.0235834

Molina, M. J. y Restrepo, D. (2018). Internet y comportamiento suicida en adolescentes: ¿cuál es la conexión? Pediatría, 51(2), 30-39. https://doi.org/10.14295/pediatr.v51i2.109

Mok, K., Jorm, A. F. y Pirkis, J. (2015). Suicide-related Internet use: A review. Australian \& New Zealand Journal of Psychiatry, 49(8). https://doi.org/10.1177/0004867415569797

Pérez, O. (2019). Uso de internet y conductas suicidas en adolescentes de 14 a 18 años en México. https://bit. ly/3onldqn 
Picardo, J., McKenzie, S. K., Collings, S. y Jenkin, G. (2020). Suicide and self-harm content on Instagram: A systematic scoping review. PLoS One, 15(9), e0238603. https://doi.org/10.1371/journal.pone.0238603

Robert, A., Suelves, J. M., Armayones, M. y Ashley, S. (2015). Internet use and suicidal behaviors: internet as a threat or opportunity? Telemedicine and e-Health, 21(4). https://doi.org/10.1089/tmj.2014.0129

Shanahan, N., Brennan, C. y House, A (2019). Self-harm and social media: Thematic analysis of images posted on three social media sites. BMJ Open, (9), e027006. https://doi.org/10.1136/bmjopen-2018-027006 


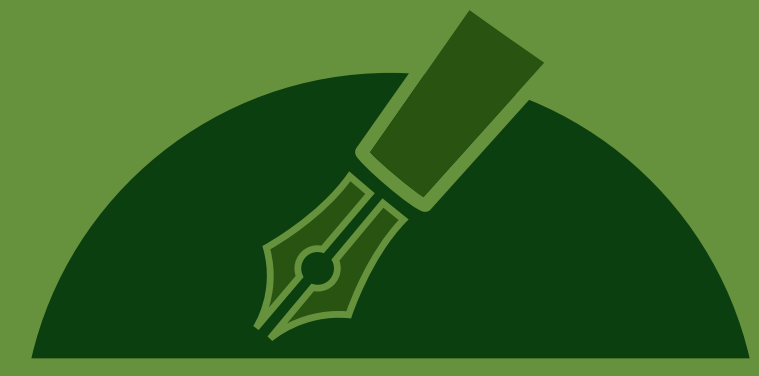

Lecturas

críticas 